\title{
Локализованные состояния $\pi$-электронов в спектре оптического поглощения высокотетраэдрического аморфного углерода
}

\author{
() М.С. Чекулаев, С.Г. Ястребов
}

Физико-технический институт им. А.Ф. Иофрфе Российской академии наук, 194021 Санкт-Петербург, Россия

E-mail: mchs89@gmail.com

Поступила в Редакцию 18 октября 2021 г.

В окончательной редакции 15 ноября 2021 r.

Принята к публикации 20 декабря 2021 r.

\begin{abstract}
Ab initio методы использованы для расчета спектра молярной экстинкции некоторых модельных кластеров, включая молекулярный гибрид $\mathrm{C}_{24} \mathrm{H}_{30}$. На основании сравнения расчета с экспериментальными спектрами основных аллотропных модификаций углерода максимум поглощения с энергией $\sim 6$ эВ в спектре поглощения высокотетраэдрического аморфного углерода отнесен к $\pi \rightarrow \pi^{*}$ оптическим переходам электронов в единичном ароматическом кольце. Краевые участки кольца ковалентно связаны с $s p^{3}$-гибридизированными атомами углерода аморфной матрицы. Проявление плеча в спектре мнимой части показателя преломления пленки высокотетраэдрического аморфного углерода, 4.6 эВ, приписывается оптическим переходам $\pi \rightarrow \pi^{*}$ кластеров, искаженных гибридным дефектом Стоуна-Уэлса, также ковалентно связанных с аморфной матрицей.
\end{abstract}

Ключевые слова: взаимодействие света с веществом, новые формы углерода, высокотетраэдрический аморфный углерод, дефект Стоуна-Уэльса.

DOI: 10.21883/FTP.2022.04.52199.9757

\section{1. Введение}

„Алмазоподобная“ разновидность аморфного углерода, получаемого в лабораторных условиях в виде аморфных тетраэдрически координированных углеродных пленок с высоким содержанием $s p^{3}$-фазы, обладает рядом свойств, присущих аморфным полупроводникам, таких как наличие запрещенной зоны и возможность изменения типа проводимости под воздействием легирования. Поэтому этот материал принято относить к группе аморфных полупроводников. В зарубежной литературе его обозначают как ta-C, где буква $\mathrm{t}$ относится к слову тетраэдрический (tetrahedral), а буква а к слову аморфный (amorphous). Этот материал представляет интерес для исследователей из-за расширения областей его применения, например при создании износо- и химически стойких покрытий. Эти свойства материала проявляются из-за высокого содержания в нем тетраэдрически координированных связей между атомами углерода. Более того, $s p^{2}$-гибридизованные кластеры (по современной терминологии - фрагменты графена) и их сети могут содержаться в его матрице [1]. Поэтому из-за слабого перекрытия областей оптической прозрачности между высокотетраэдрической матрицей и фрагментов $s p^{2}$-гибридизованных кластеров (фрагментов графена и их сетей) этот материал является едва ли не идеальной средой для их исследования методами оптической спектроскопии из-за общего интереса к новым аллотропным модификациям углерода.

Поэтому в настоящей работе приводится пример типичного спектра мнимой части показателя прелом- ления ta-C, полученный в работе [2], и проводится его интерпретация.

Поскольку, как будет показано далее, наиболее интенсивная полоса поглощения ta-C (см. [2]) практически совпадает с известной из астрофизических наблюдений полосой с максимумом $\sim 217.5 \mathrm{Hм}(\sim 5.67$ эВ $)$, в работе будут использованы результаты анализа спектра локализованных состояний $\pi$-электронов гибридного кластера $\mathrm{C}_{24} \mathrm{H}_{30}$, расчетный спектр молярной экстинкции которого лежит в пределах этой полосы. Напомним, что нами ранее в работах [3-6] методами ab initio оптимизировалась геометрия и изучалось поглощение электромагнитного излучения видимого и ультрафиолетового излучения гибридными нанокластерами разных типов, в том числе представляющими собой расположенный в центре фрагмент графена с небольшим числом ароматических колец, окруженный связанным с его боковыми сайтами фрагментом даймондена, оборванные связи которого пассивированы водородом. Кроме того, на примере дефекта Стоуна-Уэльса (СУ) [6], было рассмотрено влияние дефекта на спектр молярной экстинкции такого гибридного кластера. На основании расчетов был сделан вывод, что фрагмент графена, окруженный $s p^{3}$ матрицей, может являться кандидатом на роль поглотителя света в межзвездной среде, приводя к формированию интенсивной полосы поглощения света межзвездной средой с максимумом 217.5 нм ( 5.7 эВ). В настоящей работе мы рассчитаем укороченную структуру, содержащую такой дефект, и исследуем ее оптические свойства.

Поскольку наиболее простой кластер, $\mathrm{C}_{24} \mathrm{H}_{30}$, спектр поглощения которого наиболее близок к эксперименту, 
уже найден и исследован нами в работе [4] с помощью методов ab initio, возьмем его за основу дальнейшего анализа. В настоящей работе будет исследован механизм прикрепления к матрице с помощью боковых сайтов гибрида и выполнен расчет влияния такого закрепления на его оптические свойства для двух предельных случаев. Первый случай - когда для закрепления задействованы все боковые сайты, и второй - когда задействован только один. Для демонстрации возможности присоединения к матрице, протоны боковых сайтов замещаются углеродом, а оставшиеся оборванные связи пассивируются водородом. Будет показано, что в результате образуется $s p^{3}$-связь, моделирующая присоединение кластера к матрице. При этом система $\pi$ электронов $s p^{2}$-фрагмента графена кластера оказывается локализованной внутри матрицы, окруженная $s p^{3}$-гибридизованным углеродом. Таким образом создаются условия для геометрического конфайнмента $\pi$ электронов в матрице. Набор оптических переходов между уровнями размерного квантования изолированного фрагмента графена отвечает за спектр оптического поглощения света такими локализованными состояними $\pi$-электронов.

\section{2. Методика эксперимента}

Для исследования локализованных состояний $\pi$-электронов с использованием ab initio методов решались две задачи. Первая - оптимизация геометрии кластера $\mathrm{C}_{24} \mathrm{H}_{30}$, протон бокового сайта которого замещается углеродом, а оборванные связи пассивируются водородом. Таким образом, для выяснения возможности сопряжения с матрицей ta-C присоединим к боковому сайту кластеpa $\mathrm{C}_{24} \mathrm{H}_{30}$ дополнительную $\mathrm{C}-\mathrm{H}$-связь, моделирующую связь с матрицей. Изображение кластера $\mathrm{C}_{24} \mathrm{H}_{30}$ с присоединенной $\mathrm{C}-\mathrm{H}-$ связью представлено на рис. 1 после оптимизации геометрии, где зеленым цветом окрашен добавленный атом углерода, а желтым - водорода.

Кроме того, будет исследован кластер $\mathrm{C}_{32} \mathrm{H}_{38}$, содержащий гибридный дефект СУ. Изображение приведено на рис. 2. Бирюзовым цветом показан фрагмент кластера, содержащий дефект СУ.

Таким образом, в настоящей работе вводится новый, гибридный, тип дефекта, как это показано на рис. 2. Он формировался так же, как и классический дефект СУ, за исключением того, что после присоединения водорода к боковым сайтам часть $s p^{2}$-связей между атомами трансформировалась в $s p^{3}$.

Для расчетов использовались методы ab initio. Заметим, что подбор базиса и метода под конкретную задачу, решаемую методами ab initio, представляется довольно распространенной практикой и требует перебора значительного количества доступных комбинаций и сочетаний методов и базисов, а также сравнения результатов расчета с экспериментом для как можно более близких систем. Однако, хотя подобная процедура

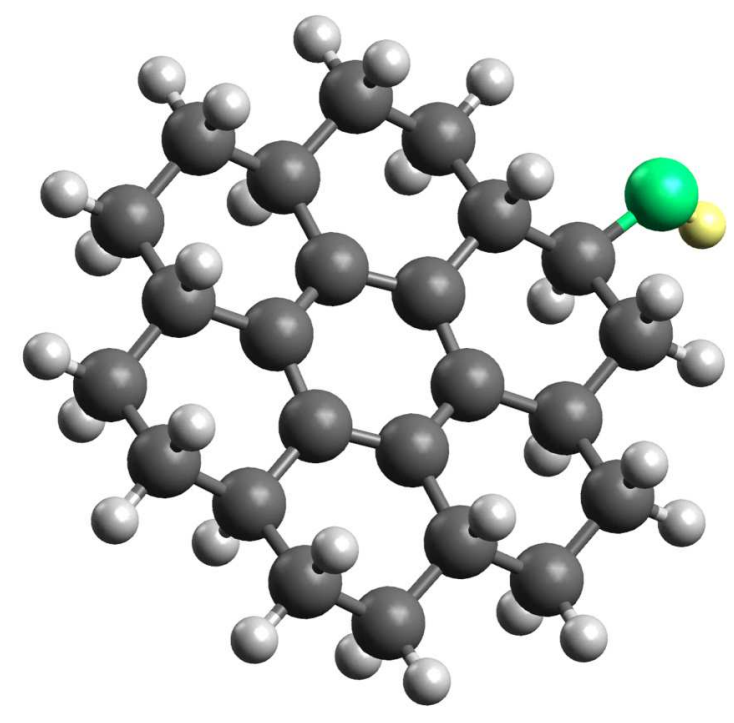

Рис. 1. Изображение кластера $\mathrm{C}_{24} \mathrm{H}_{30}$ с присоединенной СН-группой. Темно-серым и зеленым цветом помечены атомы углерода, светло-серым и желтым - водорода. Общая энергия после оптимизации геометрии равна -2536223 кДж/моль. (Цветной вариант рисунка представлен в электронной версии статьи).

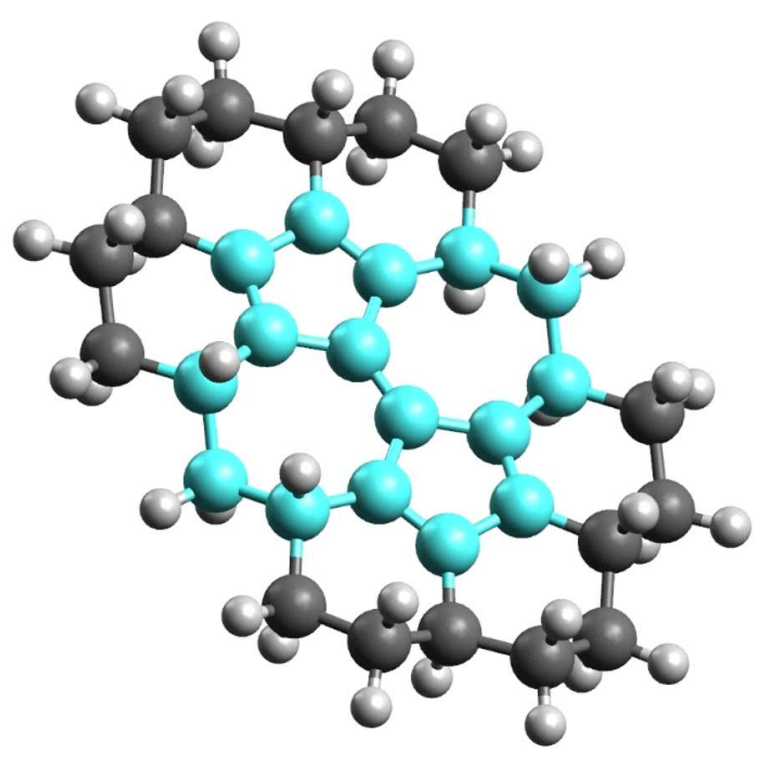

Рис. 2. Изображение кластера $\mathrm{C}_{32} \mathrm{H}_{38}$ с дефектом СУ. Темносерым и бирюзовым цветом помечены атомы углерода, светлосерым - водорода. Общая энергия после оптимизации геометрии равна -3242889 кДж/моль. (Цветной вариант рисунка представлен в электронной версии статьи).

и представляется весьма полезной, и поэтому необходимой для выполнения, но одновременно является дорогостоящей с точки зрения затрат времени как экспериментатора, так и компьютера. Действительно, помимо B3LYP, даже в рамках DFT (density functional theory, теория функционала плотности) существует множество 
методов: классические, гибридные, двойные гибриды и т.д. Каждый из них имеет свои особенности. Поэтому с целью экономии ресурсов исследователи зачастую используют литературные данные, повествующие об удачном применении тех или иных методов и базисов для решения похожих задач. В работе [7] сообщается об удачном применении обменно-корреляционного гибридного функционала B3LYP с малым базисом 3-21G(*) при оптимизации геометрии и моделирования спектральных и электрохимических свойств сложных углеродных комплексов. Поэтому в настоящей работе для оптимизации геометрии всех исследуемых кластеров и расчета их оптических свойств используются только что упомянутые обменно-корреляционный гибридный функционал и базис, по аналогии с работой [7].

Для расчетов использовался пакет GAUSSIAN 09 [8], оптимизация геометрии гибридного кластера в нашем случае выполнялась с применением алгоритма Берни [9] совместно с методом GEDIIS (geometry optimization using energy-represented direct inversion in the iterative subspace) [10]. Результаты представлены на рис. 1,2. Численные значения полной энергии молекулярного гибрида, достигнутые в ходе оптимизации в точке минимума, приведены в подписи к рис. 1,2. Знак и порядок величины этих энергий соответствуют значениям, полученным при аналогичном методе расчета для известных углеводородов (см., например, [4]). После подтверждения устойчивости геометрии методом зависящего от времени (в русскоязычной литературе - „нестационарного“) функционала плотности (time-dependent density functional theory, TD DFT) выполнялся расчет спектра молярной экстинкции. При расчете использовался описанный выше набор базисных функций.

\section{3. Результаты и обсуждение}

Обзорный спектр оптического поглощения ta-C взят из работы [2], и часть его, для актуальной области, приведена на рис. 3,4 в сравнении с расчетными данными для различных кластеров, рассматриваемых в работе.

На спектре выделяются четыре области. В области 1 поглощение увеличивается при уменьшении энергии фотона, в инфракрасной области. В области 2 при энергии фотонов, несколько превышающей 4.2 эВ, наблюдается плечо. При дальнейшем увеличении энергии в области 3 на зависимости проявляется максимум вблизи 6 эВ, с последующим падением с ростом энергии $>6$ эВ. При дальнейшем увеличении энергии спад сменяется ростом в области, которая отмечена цифрой 4 на рисунке.

Поскольку речь идет об углероде, полезно его сравнить со спектрами основных аллотропных модификаций - алмаза и графита, мнимая часть которых представлена на рис. 5. Обращаем внимание на рост мнимой части в низкоэнергетической части спектра, который повторяет тренд для графита. В бесщелевом графите этот рост происходит отчасти из-за оптических

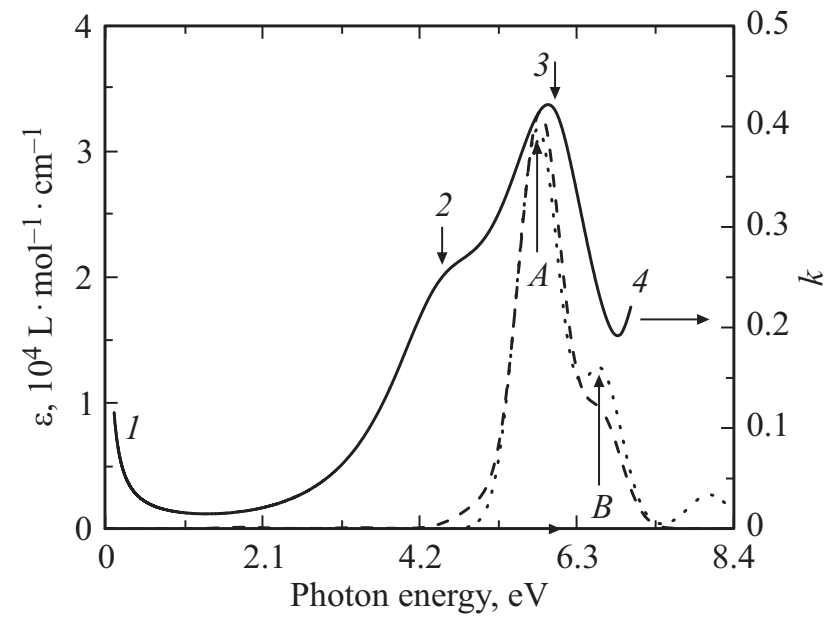

Рис. 3. Расчетный спектр молярной экстинкции для фрагмента c $\mathrm{C}_{24} \mathrm{H}_{30}$, - пунктирная линия. Расчетный спектр молярной экстинкции для фрагмента с $\mathrm{C}_{24} \mathrm{H}_{30}$ и для фрагмента с $\mathrm{C}_{24} \mathrm{H}_{30} \mathrm{c}$ присоединенной С-H-группой показаны соответственно пунктирной и штриховой линиями. Спектр мнимой части показателя преломления ta-C [2] показан сплошной линией.

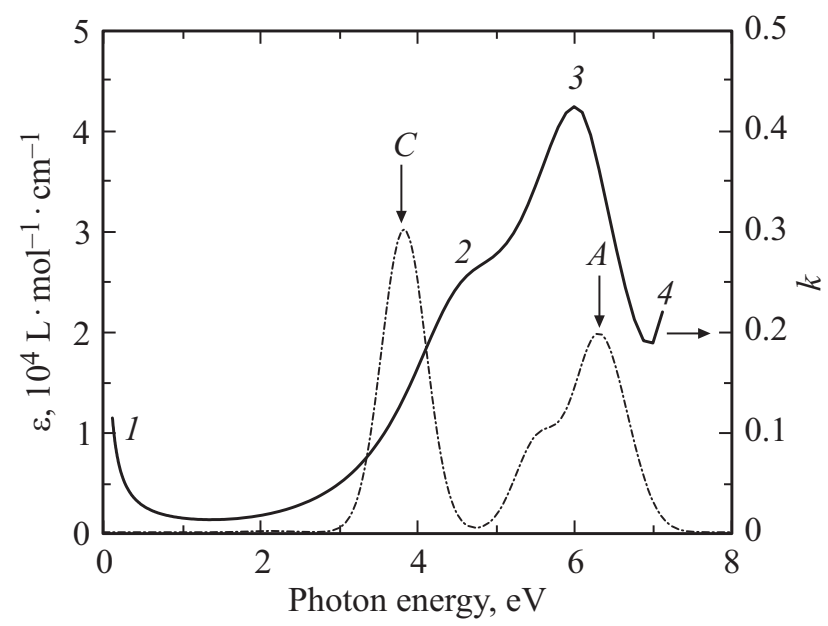

Рис. 4. Расчетный спектр молярной экстинкции для фрагмента с дефектом типа СУ - штрихпунктирная линия. Спектр мнимой части показателя преломления ta-C [2] показан сплошной линией.

переходов $\pi \rightarrow \pi^{*}$ и благодаря наличию свободных электронов в зоне проводимости. Также можно отметить общность в области 2, между ходом зависимости для ta-C и графита. Если в случае ta-C на зависимости наблюдается плечо, то для графита - максимум мнимой части. В случае графита этот максимум формируется переходами $\pi \rightarrow \pi^{*}$. Учитывая родственность обоих материалов, можно приписать наличие плеча спектра ta-C длинным фрагментам графена, внедренным в аморфную сеть атомов, связанных $s p^{3}$-гибридизацией. Возможно также, что эта сеть связана с внутренней и внешней поверхностями образца по аналогии с графитизированной поверхностью [111] алмаза [11]. Основное отличие 


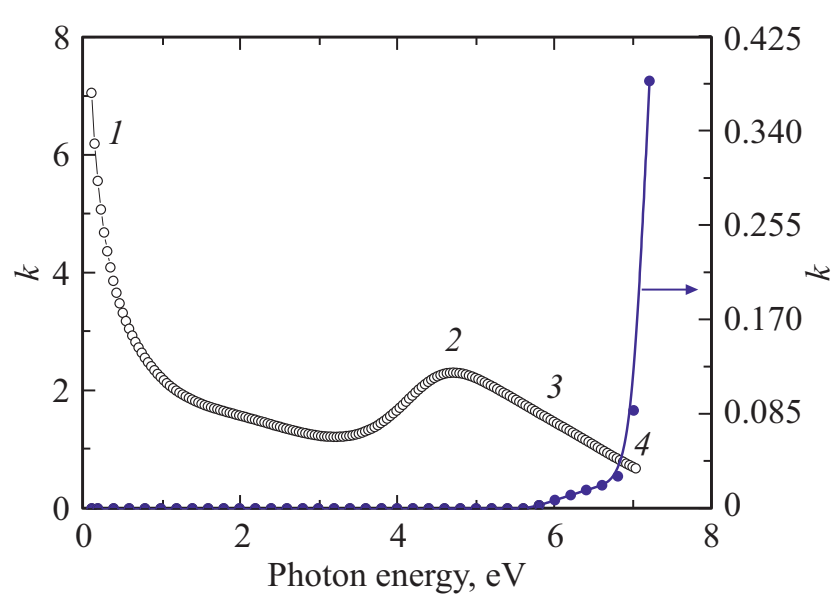

Pис. 5. Сплошные кружки - спектр мнимой части показателя преломления алмаза (эксперимент) [12]. Пустые кружки спектр мнимой части показателя преломления графита (эксперимент) для направления электрического поля электромагнитной волны, параллельного базальной плоскости [13].

спектров графита и алмаза от ta-C, как видно из рисунка, состоит в полосе поглощения, занимающей область 3. Далее мы покажем, что эту полосу поглощения можно также приписать оптическим переходам $\pi \rightarrow \pi^{*}$ фрагмента графена, электроны которого, в силу малости фрагмента, подвергаются геометрическому конфайнменту.

Для перпендикулярного направления в актуальном спектральном диапазоне поглощение мало по сравнению с представленным на рисунке.

Рассмотрим гибридный кластер, состоящий из единичного ароматического кольца, инкапсулированного фрагментом даймондена, как это описано выше. Расчетный спектр молярной экстинкции для такого кластера с формулой $\mathrm{C}_{24} \mathrm{H}_{30}$ приведен на рис. 3. Максимумы поглощения в актуальной области спектра отмечены вертикальными стрелками и буквами $A$ и $B$, а также вертикальными стрелками, направленными вверх. Заметим, что энергия, соответствующая наиболее интенсивному максимуму, равна $\sim 5.9$ эВ, а энергия, соответствующая менее интенсивному максимуму, равна $\sim 6.7$ эВ. Такой фрагмент графена, содержащий одно ароматическое кольцо, может существовать в исследуемой разновидности ta-C, связанный с матрицей боковыми сайтами. В данном случае водород краевых сайтов кластера моделирует его соединение с атомами матрицы, преимушественно связанными друг с другом $s p^{3}$-типом гибридизации. При этом проявление интенсивной полосы поглощения на спектре ta-C, отмеченной цифрой 3 , может быть связано с оптическим поглощением света $\pi$ электронной системой единичного ароматического кольца, претерпевающей геометрический конфайнмент (оптические переходы $\pi \rightarrow \pi^{*}$, где буквами $\pi$ и $\pi^{*}$ обозначены соответственно локализованные состояния, занятые электронами, и свободные от них).
Для исследования сохранения устойчивости гибридного кластера при замене водорода бокового сайта на углерод к нему была добавлена группа $\mathrm{C}-\mathrm{H}$, как это показано на рис. 1. Как видно из рисунка, кластер сохраняет устойчивость. Соответствующий расчет оптических свойств в сравнении со спектром мнимой части показателя преломления ta-C приведен на рис. 3. Видно, что в случае присоединения гибридного кластера к матрице через единичный боковой сайт спектр молярной экстинкции модифицируется незначительно.

Также удается выяснить влияние гибридного дефекта типа СУ на спектр локализованных состояний $\pi$ электронов. Подобные расчеты предпринимались ранее [6], было получено, что дефект СУ приводит к образованию особенности в области плеча на спектре, подобном определенному для ta-C. Соответствующий заявленной выше конфигурации атомов, показанной на рис. 2, спектр молярной экстинкции представлен на рис. 4. По сравнению с результатами расчета, представленными выше, для кластера, искаженного дефектом СУ, происходит сдвиг максимума полосы $A$ в область более высоких энергий, 6.28 эВ, сопровождающийся уменьшением ее интенсивности. Также возникает полоса поглощения 3.83 эВ, проявляющаяся в области плеча спектра мнимой части показателя преломления ta-C (обозначена на рис. 4 буквой $C$ ). Область плеча, проявляющегося на спектре $\mathrm{t}$-aC, помечена цифрой 2 на рис. 4, 5 .

Итак, при проведении сравнения расчетов, выполненных методом $a b$ initio, удалось установить связь между спектром мнимой части ta-C и расчетными коэффициентами молярной экстинкции гибридных кластеров двух типов, содержащих одно ароматическое кольцо, и кластера, содержащего дефект, подобный дефекту СУ. Все расчеты выявляют полосу поглощения с максимумом при энергии $\sim 6$ эВ, что совпадает с наиболее интенсивной особенностью спектра ta-С. Расчет для кластера, содержащего дефект, подобный дефекту СУ, позволяет выявить полосу поглощения с максимумом $\sim 3.83$ эВ, расположенную вблизи плеча спектра ta-C.

Для объяснения роста поглощения с уменьшением энергии фотонов в ИК области спектра выше мы использовали сходство этого участка спектра с аналогичным, измеренным для графита, и предположили, что его природа связана с образованием протяженных фрагментов графена. Для дальнейшего исследования такого поведения мнимой части уточним модель.

Выше мы сравнивали безразмерную мнимую часть показателя преломления с размерным спектром коэффициента молярной экстинкции. Размерная часть, кроме прочего, содержит обратные сантиметры, т.е. является аналогом коэффициента поглощения $\alpha$ :

$$
\alpha=\frac{4 \pi k}{\lambda}
$$

Только в этом месте статьи $\pi$ это известная математическая константа, $\lambda$ - длина волны электромагнитного 


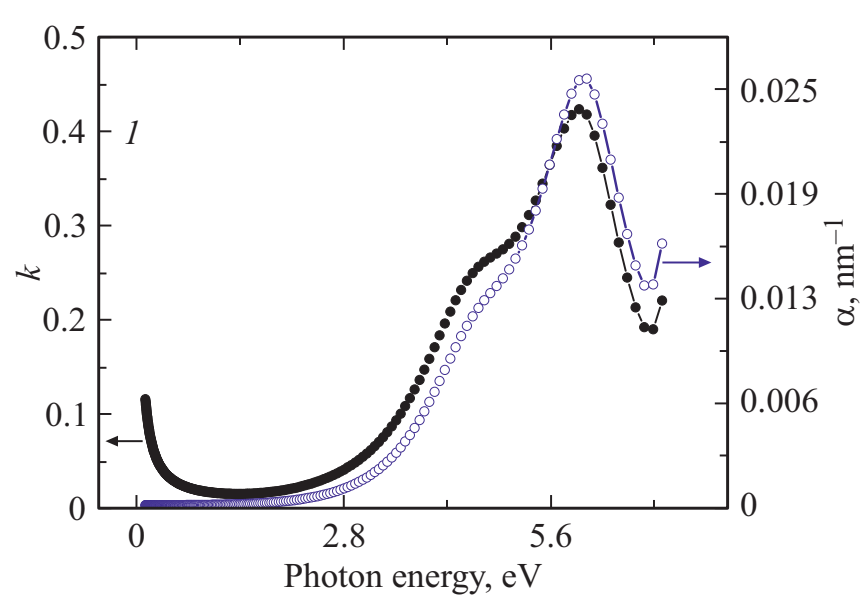

Рис. 6. Сравнение спектров мнимой части показателя преломления и коэффициента поглощения ta-C.

излучения, $k$ - мнимая часть показателя преломления. На рис. 6 приведено сравнение $k$ и $\alpha$.

Видно, что, кроме абсолютных значений величин, зависимости рис. 6 отличаются только в низкоэнергетической части спектра, где $\alpha$ в отличии от $k$ не возрастает с уменьшением энергии. Положения максимумов и плеча на спектре $\alpha$ не претерпевают изменений. Поэтому проявление плеча на спектре мнимой части показателя преломления пленки ta-C, 4.6 эВ, можно также связать с оптическим переходом $\pi \rightarrow \pi^{*}$ в дефектных гибридных кластерах, ковалентно связанных с аморфной матрицей.

\section{4. Заключение}

С использованием методов ab initio выполнены:

1) оптимизация геометрии гибридных кластеров $\mathrm{C}_{24} \mathrm{H}_{30}$ с присоединенной СН-группой и $\mathrm{C}_{32} \mathrm{H}_{38}$, искаженного дефектом СУ;

2) расчет спектров молярной экстинкции.

На основании выполненных расчетов сделан вывод об устойчивости этих кластеров.

Выполненное сравнение расчета с экспериментальным спектром оптического поглощения ta-C позволило интерпретировать максимум с энергией $\sim 6$ эВ вклад оптических переходов $\pi \rightarrow \pi^{*}$ единичных ароматических колец, ковалентно связанных с аморфной матрицей.

„Плечо“ на спектре в области $\sim 4.6$ эВ можно также приписать вкладу оптических переходов $\pi \rightarrow \pi^{*}$ гибридных кластеров, подобных $\mathrm{C}_{32} \mathrm{H}_{38}$, которые искажены дефектами типа СУ.

\section{Благодарности}

Авторы благодарны Е.Ю. Тупикиной - ассистенту кафедры физической химии СПбГУ за многочисленные полезные обсуждения и консультации по практическому применению пакета GAUSSIAN 09.

\section{Конфликт интересов}

Авторы данного исследования заявляют об отсутствии конфликта интересов.

\section{Список литературы}

[1] C.T. Toh, H. Zhang, J. Lin, A.S. Mayorov, Y. Wang, C.M. Orofeo, D.B. Ferry, H. Andersen, N. Kakenov, Z. Guo, I.H. Abidi, H. Sims, K. Suenaga, S.T. Pantelides, B. Özyilmaz. Nature, 577, 199 (2020).

[2] J.I. Larruquert, L.V. Rodríguez-de Marcos, J.A. Méndez, P.J. Martin, A. Bendavid. Opt. Express, 21, 27537 (2013).

[3] М.С. Чекулаев, С.Г. Ястребов. Письма ЖТФ, 45 (8), 47 (2019).

[4] М.С. Чекулаев, С.Г. Ястребов. Письма ЖТФ, 47 (19), 44 (2020).

[5] М.С. Чекулаев, С.Г. Ястребов. Письма ЖТФ, 47 (4), 19 (2021).

[6] М.С. Чекулаев, С.Г. Ястребов. Письма ЖТФ 47 (24), 34 (2021)

[7] M.E. Zandler, F. D’Souza. Comptes Rendus Chimie, 9(7-8), 960 (2006).

[8] M.J. Frisch, G.W. Trucks, H.B. Schlegel, G.E. Scuseria, M.A. Robb, J.R. Cheeseman, G. Scalmani, V. Barone, B. Mennucci, G.A. Petersson, H. Nakatsuji, M. Caricato, X. Li, H.P. Hratchian, A.F. Izmaylov, J. Bloino, G. Zheng, J.L. Sonnenberg, M. Hada, M. Ehara, K. Toyota, R. Fukuda, J. Hasegawa, M. Ishida, T. Nakajima, Y. Honda, O. Kitao, H. Nakai, T. Vreven, J.A.J. Montgomery, J.E. Peralta, F. Ogliaro, M. Bearpark, J.J. Heyd, E. Brothers, K.N. Kudin, V.N. Staroverov, R. Kobayashi, J. Normand, K. Raghavachari, A. Rendell, J.C. Burant, S.S. Iyengar, J. Tomasi, M. Cossi, N. Rega, J.M. Millam, M. Klene, J.E. Knox, J.B. Cross, V. Bakken, C. Adamo, J. Jaramillo, R. Gomperts, R.E. Stratmann, O. Yazyev, A.J. Austin, R. Cammi, C. Pomelli, J.W. Ochterski, R.L. Martin, K. Morokuma, V.G. Zakrzewski, G.A. Voth, P. Salvador, J.J. Dannenberg, S. Dapprich, A.D. Daniels, Ö. Farkas, J.B. Foresman, J.V. Ortiz, J. Cioslowski, D.J. Fox. Gaussian, 09, (2013).

[9] H.B. Schlegel. Adv. Chem. Phys., 67, 249 (2007).

[10] X. Li, M.J. Frisch. J. Chem. Theory Comput., 2, 835 (2006).

[11] O.A. Shenderova, D.M. Gruen. Ultrananocrystalline Diamond: Synthesis, Properties and Applications (Oxford, Elsevier, 2012) chap. 7, p. 181.

[12] H.R. Philipp, E.A. Taft. Phys. Rev., 127, 159 (1962).

[13] A.B. Djurišić, E.H. Li. J. Appl. Phys., 85, 7404 (1999).

Редактор Г.А. Оганесян 


\section{Localised $\pi$-electron states in the optical absorption spectrum of tetrahedrally coordinated amorphous carbon}

M.S. Chekulaev, S.G. Yastrebov

loffe Institute,

194021 St. Petersburg, Russia

Abstract The paper presents result of ab initio methods exploiting to calculate the molar extinction spectrum of some model clusters, the molecular hybrid $\mathrm{C}_{24} \mathrm{H}_{30}$ included. Based on the comparison of the calculation with the experimental spectra of the main allotropic modifications of carbon, the absorption maximum with an energy of $\sim 6 \mathrm{eV}$ in the absorption spectrum of highly tetrahedral amorphous carbon (ta-C) attributed to the $\pi \rightarrow \pi^{*}$ optical transitions of electrons in a single aromatic ring. The edge sites of the ring covalently bonded with the $s p^{3}$ hybridised carbon atoms of the amorphous matrix. The manifestation of a shoulder in the spectrum of the imaginary part of the refractive index of a film of highly tetrahedral amorphous carbon (ta-C), $4.6 \mathrm{eV}$, is assigned to $\pi \rightarrow \pi^{*}$ optical transitions of clusters distorted with hybrid Stone-Wallace-like defect, also covalently bonded with the amorphous matrix. 